\title{
Matchings avoiding partial patterns and lattice paths
}

\author{
Vít Jelínek \\ Department of Applied Mathematics, Charles University, \\ Malostranské náměstí 25, Prague, Czech Republic. \\ jelinek@kam.mff.cuni.cz \\ Nelson Y. Li \\ Center for Combinatorics, LPMC, Nankai University, \\ 300071 Tianjin, P.R. China \\ nelsonli@eyou.com \\ Toufik Mansour \\ Department of Mathematics, University of Haifa, \\ 31905 Haifa, Israel. \\ Center for Combinatorics, LPMC, Nankai University, \\ 300071 Tianjin, P.R. China \\ toufik@math.haifa.ac.il \\ Sherry H. F. Yan \\ Center for Combinatorics, LPMC, Nankai University, \\ 300071 Tianjin, P.R. China \\ huifangyan@eyou.com
}

Submitted: May 17, 2006; Accepted: Sep 29, 2006; Published: Oct 19, 2006

Mathematics Subject Classification: 05A05, 05C30.

\begin{abstract}
In this paper, we consider matchings avoiding partial patterns 1123 and 1132. We give a bijection between 1123-avoiding matchings with $n$ edges and nonnegative lattice paths from $(0,2)$ to $(2 n, 0)$. As a consequence, the refined enumeration of 1123-avoiding matchings can be reduced to the enumeration of certain lattice paths. Another result of this paper is a bijection between 1132-avoiding matchings with $n$ edges and lattice paths from $(0,0)$ to $(2 n, 0)$ starting with an up step, which may go under the $x$-axis.
\end{abstract}

\section{Introduction}

A matching on a set $[2 n]=\{1,2, \ldots, 2 n\}$ is a partition of $[2 n]$ of the type $(2,2, \ldots, 2)$, or equivalently, a graph in which every vertex has degree one. There are many ways to 
represent a matching. It can be displayed by drawing $2 n$ points in the plane lying on a horizontal line, and connecting them by $n$ arcs, each arc connecting two of the points and lying above the points; such representation is called the linear representation of the matching [1]. An edge $(i, j)$ is drawn as an arc between the nodes $i$ and $j$ above the horizontal line, where the vertices $i$ and $j$ are called the initial point and the end point, respectively. An edge $e=(i, j)$ is always written in such a way that $i<j$. Let $e=(i, j)$ and $e^{\prime}=\left(i^{\prime}, j^{\prime}\right)$ be two edges of a matching $M$; we say that $e$ crosses $e^{\prime}$ if they intersect each other, in other words, if $i<i^{\prime}<j<j^{\prime}$. Otherwise, $e$ and $e^{\prime}$ are said to be noncrossing. Similarly, if $i<i^{\prime}<j^{\prime}<j$, we say that $e^{\prime}$ is nested below $e$. The set of all the matchings on $[2 n]$ is denoted by $\mathcal{M}_{n}$. Note that $\left|\mathcal{M}_{n}\right|=(2 n-1) ! !=1 \cdot 3 \cdot 5 \cdots(2 n-1)$.

In this paper, we also use the representation of a matching $M$ of $n$ edges by a sequence of length $2 n$ on the set $\{1,2, \ldots, n\}$ such that each element $i(1 \leq i \leq n)$ appears exactly twice, and the first occurrence of the element $i$ precedes that of $j$ if $i<j$. Such a representation is called the canonical sequential form [8]. In fact, the canonical sequential representation of a matching can be obtained from its linear representation by labelling the endpoints of each arc in the order of the appearance of its initial points using the set $[n]$. For example, the matching in Figure 1 can be represented by 123123. For a matching represented by its canonical sequential form and for $j \in[n]$, we use the term edge $j$ to refer to the edge that connects the two vertices labeled $j$.

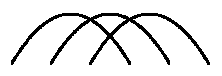

Figure 1: The matching 123123.

A matching with canonical sequential form $M$ is called separable, if $M=12 \ldots n M^{\prime}$ where $M^{\prime}$ is a permutation of $1,2, \ldots, n$. Note that a matching is separable if and only if all the initial points of edges appear to the left of any end point.

Let $\pi=\pi_{1} \pi_{2} \ldots \pi_{k}$ and $\tau=\tau_{1} \tau_{2} \ldots \tau_{k}$ be two sequences. We say that $\pi$ and $\tau$ are order-isomorphic, if for each $1 \leq i, j \leq k$ we have $\pi_{i}<\pi_{j}$ if and only if $\tau_{i}<\tau_{j}$. We say that a matching $M$ contains an occurrence of $\pi$ if there is a subsequence in the canonical sequential form of $M$ which is order-isomorphic to $\pi$. In such a context, $\pi$ is usually called a pattern. If $M$ does not contain $\pi$, we say that $M$ avoids $\pi$, or $M$ is $\pi$-avoiding. The set of all $\pi$-avoiding matchings on $[2 n]$ is denoted $\mathcal{M}_{n}(\pi)$.

Pattern avoiding matchings have been studied by de Médicis and Viennot [12], de Sainte-Catherine [13], Gessel and Viennot [5], Gouyou-Beauchamps [6, 7], Stein [15], Touchard [16], and recently by Klazar [8, 9, 10], Chen, Deng, Du, Stanley and Yan [2], Chen, Mansour, Yan [3].

In this paper, we get a refined enumeration of 1123-avoiding matchings according to the positions of edge $n$ by providing a bijection between 1123-avoiding matchings with $n$ edges and nonnegative lattice paths from $(0,2)$ to $(2 n, 0)$. As a consequence, the enumeration can be reduced to the enumeration of lattice paths according to the positions of the first two down steps. Another result of this paper is a bijection between 1132-avoiding matchings with $n$ edges and lattice paths from $(0,0)$ to $(2 n, 0)$ starting with an up step. 


\section{1123-avoiding matchings and lattice paths}

In this section, we consider the enumeration of 1123-avoiding matchings. The clear structure leads to a bijection from the set of 1123-avoiding matchings to the set of nonnegative lattice paths from $(0,2)$ to $(2 n, 0)$. The refined enumeration of 1123 -avoiding matchings according to the positions of edge $n$ can be reduced to the enumeration of lattice paths according to the positions of the first two down steps.

Let $\mathbb{Z}$ denote the set of all integers; a lattice path is a path on the integer lattice $\mathbb{Z} \times \mathbb{Z}$ composed of up steps which connect a point $(x, y)$ with a point $(x+1, y+1)$, and down steps connecting $(x, y)$ with $(x+1, y-1)$. A nonnegative lattice path is a lattice path never going below the $x$-axis. A nonnegative lattice path connecting $(0,0)$ with $(2 n, 0)$ is called a Dyck path. The number of Dyck paths from $(0,0)$ to $(2 n, 0)$ is given by the $n$-th

Catalan number $c_{n}=\frac{1}{n+1}\left(\begin{array}{c}2 n \\ n\end{array}\right)$. Throughout this section, all the lattice paths are assumed to be nonnegative lattice paths, even if the nonnegativity is not explicitly stated.

We start with a lemma describing the structure of 1123-avoiding matchings.

Lemma 2.1. A matching $M$ with $n$ edges is 1123-avoiding if and only if it satisfies the following three conditions:

1. There is no end point of an edge before the initial point of the edge $n-1$. In other words, after we erase the two instances of letter $n$ from the canonical sequential form of $M$, the resulting sequence of length $2 n-2$ can be represented as

$$
1,2,3, \ldots, n-1, \sigma_{1}, \sigma_{2}, \ldots, \sigma_{n-1}
$$

where $\sigma_{1}, \ldots, \sigma_{n-1}$ is a permutation of order $n-1$.

2. The permutation $\sigma$ defined above is a 123-avoiding permutation on the set $[n-1]$.

3. The edges ending before the end point of edge $n$ do not cross each other.

Proof. First, we observe that the three conditions are necessary. Assume that $M$ is a 1123-avoiding matching. $M$ satisfies the first condition, since if an edge $k$ ended before the initial point of edge $n-1$, then $M$ would contain the subsequence $k, k, n-1, n$, which is isomorphic to the forbidden pattern. $M$ satisfies the second condition as well, since if $\sigma$ contained the subsequence $i j k$ for some $i<j<k$, then $M$ would contain $i i j k$, which is forbidden. Lastly, to see that the third condition holds, note that if two crossing edges $i<j$ ended before edge $n$, then $M$ would contain the forbidden subsequence iijn. We conclude that every 1123-avoiding matching satisfies the three conditions.

To prove the converse, let $M$ be a matching satisfying the three conditions and let us assume, for contradiction, that $M$ contains a subsequence $\tau=i i j k$, with $i<j<k$. By the first condition, the occurrence of $j$ in $\tau$ corresponds to an end point of edge $j$, because the initial point of $j$ cannot occur after the end point of $i$. This shows, in fact, that the edges $i$ and $j$ must cross each other. If $k<n$, then the last three elements of $\tau$ belong to $\sigma$, which contradicts the second condition of the lemma. On the other hand, if $k=n$, then the two edges $i$ and $j$ contradict the third condition.

This concludes the proof. 
By Lemma 2.1, a 1123-avoiding matching $M$ is fully described by a permutation $\sigma$ of order $n-1$ and the positions where the initial point and the end point of edge $n$ are inserted. To state this observation rigorously, let us introduce some more terminology. Let $\sigma$ be a permutation of order $n-1$, represented as a sequence $\sigma_{1}, \sigma_{2}, \ldots, \sigma_{n-1}$. The $k$-th gap of $\sigma$ is the position between $\sigma_{k-1}$ and $\sigma_{k}$; the first gap is the position before $\sigma_{1}$ and the $n$-th gap is the position after $\sigma_{n-1}$. For a permutation $\pi$ of order $m$, the initial run of $\pi$ denotes the maximal initial decreasing sequence in $\pi$; in other words, $\pi_{1}, \pi_{2}, \ldots, \pi_{j}$ is the initial run, if $\pi_{1}>\pi_{2}>\cdots>\pi_{j}$ and either $\pi_{j}<\pi_{j+1}$ or $j=m$.

Observe that by Lemma 2.1, the canonical sequential forms of 1123-avoiding matchings with $n$ edges are exactly the sequences obtained in the following way: we choose $\sigma$ to be an arbitrary 123-avoiding permutation of order $n-1$, and we let $m$ denote the length of its initial run. We then insert two occurrences of $n$ into any of the first $m+1$ gaps of $\sigma$, possibly inserting both occurrences of $n$ into the same gap. Note that there are $\left(\begin{array}{c}m+2 \\ 2\end{array}\right)$ ways to perform the insertion. After the insertion, we prefix the whole sequence with $1,2, \ldots, n-1$ to obtain a canonical sequential form of a 1123-avoiding matching.

Our next goal is to find closed form expressions enumerating the 1123-avoiding matchings of a given size. This is achieved by constructing a bijection to a suitable set of lattice paths. The first ingredient of our argument is a well known bijection from the set of 123avoiding permutations on $[m]$ to Dyck paths of length $m$, which was given by Krattenthaler [11]. Here we give a description of the bijection without proof. Given a 123-avoiding permutation $\pi$ of order $m$, let $r_{1}, r_{2}, \ldots, r_{s}$ be the right-to-left maxima of $\pi$; in other words, $r_{1}=\pi_{m}$ and $r_{i}$ is the last element of $\pi$ that occurs before $r_{i-1}$ and is greater than $r_{i-1}$ (so that $r_{s}=m$ ). Then $\pi$ can be decomposed into $\omega_{s} r_{s} \omega_{s-1} r_{s-1} \ldots \omega_{2} r_{2} \omega_{1} r_{1}$, where each $\omega_{i}$ is a decreasing sequence of integers and all the elements of $\omega_{i}$ are smaller than those of $\omega_{i+1}$. Then the corresponding Dyck path is constructed by encoding each $\omega_{i}$ with $\left|\omega_{i}\right|+1$ up steps and each $r_{i}$ with $r_{i}-r_{i-1}$ down steps (taking $r_{0}=0$ ). From the bijection, we observe that if $\pi_{j}=m$ (and hence $\left|\omega_{s}\right|=j-1$ ) then the corresponding Dyck path starts with $j$ up steps, followed by a down step.

In order to obtain the bijection mapping the 1123-avoiding matchings to lattice paths from $(0,2)$ to $(2 n, 0)$, we need to consider the following two sets:

$$
\begin{aligned}
& A_{j}(m)=\left\{\sigma \in S_{m}(123) ; \sigma \text { has an initial run of length } j\right\}, \\
& B_{j}(m)=\left\{\pi \in S_{m}(123) ; \pi_{j}=m\right\},
\end{aligned}
$$

where $S_{m}(123)$ is the set of 123 -avoiding permutations on the set $[m]$. We aim to find a bijection between these two sets.

Lemma 2.2. For every $j \in[m]$, there is a bijection $\rho$ from $A_{j}(m)$ to $B_{j}(m)$.

Proof. Let $\sigma \in A_{j}(m)$. We consider the following two cases:

Case a. $\sigma_{1}=m$. In this case, we define $\pi=\rho(\sigma)$ as follows:

- $\left(\pi_{1}, \pi_{2}, \ldots, \pi_{j-1}\right)=\left(\sigma_{2}, \sigma_{3}, \ldots, \sigma_{j}\right)$

- $\pi_{j}=m$ 
- $\pi_{k}=\sigma_{k}$ for every $k>j$

This maps $\sigma$ to $\pi \in B_{j}(m)$, where $\pi$ has the additional property that if we delete $\pi_{j}$ from $\pi$, the resulting permutation of order $m-1$ will have the initial run of length $j-1$.

Case b. $\quad \sigma_{1}<m$. Since $\sigma$ has initial run of length $j$, this implies that $\sigma_{j+1}=m$. This is because if we had $\sigma_{k}=m$ for some $k>j+1$, then the triple $\sigma_{j}, \sigma_{j+1}, \sigma_{k}$ would form the forbidden configuration. We now define $\pi=\rho(\sigma)$ as follows:

- $\pi_{j}=\sigma_{j+1}$

- $\pi_{j+1}=\sigma_{j}$

- $\pi_{k}=\sigma_{k}$ for every $k \notin\{j, j+1\}$

This maps $\sigma$ to $\pi \in B_{j}(m)$, where $\pi$ has the additional property that after deleting $\pi_{j}$, the initial run of the remaining permutation has length at least $j$, because it contains the initial run of $\sigma$.

The two cases above cover all possibilities. It can be easily checked that they define the required bijection.

In a lattice path, a step is said to be $k$ positioned if it is the $k$-th step from left to right.

Theorem 2.3. There is a bijection between the set of 1123-avoiding matchings with $n$ edges and the set of lattice paths from $(0,2)$ to $(2 n, 0)$. Furthermore, if the two occurrences of symbol $n$ appear at positions $n-1+k$ and $n+l$ of the canonical sequential form of the matching, with $1 \leq k \leq l$, and if after the deletion of edge $n$, the resulting matching can be represented as $12 \ldots n-1 \sigma$, where $\sigma$ has initial run of length $j$, then the first three down steps of the corresponding lattice path are $k, l+1$ and $j+3$ positioned.

Proof. Let $M$ be a 1123-avoiding matching with $n$ edges, whose canonical sequential form, after deleting edge $n$, can be represented as $12 \ldots n-1 \sigma$ with $\sigma \in A_{j}(n-1)$. Let $\pi=\rho(\sigma) \in B_{j}(n-1)$ and let $P$ be the Dyck path corresponding to $\pi$. Since $\pi \in B_{j}(n-1)$, the first down step of $P$ is $j+1$ positioned, that is, $P$ starts with $j$ up steps. If the initial point and end point of edge $n$ in $M$ are inserted into gap $k$ and gap $l$ of $\sigma$, we add one down step immediately before the $k$ positioned step and another down step before the $l$ positioned step of $P$ (note that the two added steps appear before any down step of $P$ ). Then we move the obtained lattice path two units up to obtain a new lattice path which is running from $(0,2)$ to $(2 n, 0)$. Clearly, the first three down steps of this path are $k$, $l+1$ and $j+3$ positioned, as claimed.

Conversely, assume that we are given a lattice path from $(0,2)$ to $(2 n, 0)$ whose first three down steps have positions $k, l+1$ and $j+3$, respectively. Clearly $1 \leq k \leq l \leq j+1$. Since the lattice path is nonnegative, it must have at least one up step among the first three steps, hence $j \geq 1$.

We now delete the first two down steps of this path, obtaining a Dyck path with $2 n-2$ steps, whose first down step is $j+1$ positioned. Let $\pi$ be the permutation corresponding 
to this Dyck path and $\sigma=\rho^{-1}(\pi)$. The initial run of $\sigma$ has length $j$. Then we get a matching on the set $[2 n]$ by inserting the edge $n$ into the gaps $k$ and $l$ of $\sigma$. Hence the two appearances of $n$ are at positions $n-1+k$ and $n+l$ of the canonical sequential form of the corresponding matching.

An example of the bijection between 123455643612 and the lattice path from $(0,2)$ to $(12,0)$ is shown in Figure 2.

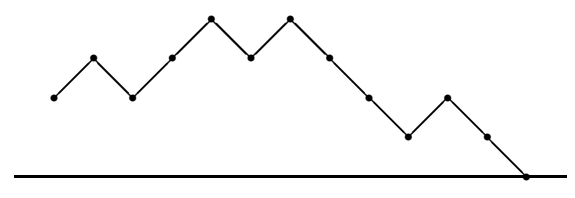

Figure 2: The bijection between a 1123-avoiding matching and a lattice path.

By standard results (see, e.g. [14, Corollary 6.2.3 and Exercise 6.20] or [4]), the number of lattice paths from $(0,0)$ to $(x, y)$ is equal to $\frac{2 y+2}{x+y+2}\left(\begin{array}{c}x \\ (x-y) / 2\end{array}\right)$. This allows us to obtain explicit formulas for several classes of 1123-avoiding matchings.

\section{Corollary 2.4.}

- The number of 1123-avoiding matchings with n edges is equal to the number of lattice paths from $(0,2)$ to $(2 n, 0)$, which is equal to

$$
\frac{3}{n+2}\left(\begin{array}{c}
2 n \\
n-1
\end{array}\right) \text {. }
$$

- For every $k \geq 1$, the number of 1123-avoiding matchings with $n$ edges such that the first occurrence of the symbol $n$ appears at the position $n-1+k$ in its canonical sequential form is equal to the number of lattice paths from $(0,2)$ to $(2 n, 0)$ whose first down step is $k$ positioned, which is equal to the number of lattice paths from $(0,0)$ to $(2 n-k, k)$, that is,

$$
\frac{k+1}{n+1}\left(\begin{array}{c}
2 n-k \\
n-k
\end{array}\right) .
$$

- For every $l \geq k \geq 1$, the number of 1123-avoiding matchings with $n$ edges such that the two occurrences of the symbol $n$ appear at the positions $n-1+k$ and $n+l$ is equal to the number of lattice paths from $(0,2)$ to $(2 n, 0)$ whose first two down steps are $k$ and $l+1$ positioned, which is equal to the number of lattice paths from $(0,0)$ to $(2 n-l-1, l-1)$, that is,

$$
\frac{l}{n}\left(\begin{array}{c}
2 n-l-1 \\
n-l
\end{array}\right)
$$




\section{$3 \quad$ 1132-avoiding matchings and lattice paths}

In this section, our goal is to show that $\mathcal{M}_{n}(1132)$ has cardinality $\frac{1}{2}\left(\begin{array}{c}2 n \\ n\end{array}\right)$ by providing a bijection between the set $\mathcal{M}_{n}(1132)$ and the set of lattice paths from $(0,0)$ to $(2 n, 0)$ starting with an up step. The lattice paths considered in this section are not necessarily nonnegative, that is, the paths may go below the $x$-axis. We will represent the lattice paths as sequences of symbols $U$ and $D$, which correspond to up steps and down steps, respectively.

We say that a matching on $[2 n]$ is trivial if its canonical sequential form is $112233 \ldots n n$. We will first show that the number of separable 1132-avoiding matchings with $n$ edges is the $n$-th Catalan number by providing a bijection from the set of separable 1132-avoiding matchings with $n$ edges to the set of Dyck paths from $(0,0)$ to $(2 n, 0)$. Next, we will define a bijection from the set of 1132-avoiding matchings with $n$ edges to the set of lattice paths from $(0,0)$ to $(2 n, 0)$ starting with an up step.

Note that a matching is 1132-avoiding, if and only if no three edges $i<j<k$ have the relative positions illustrated by Figure 3.
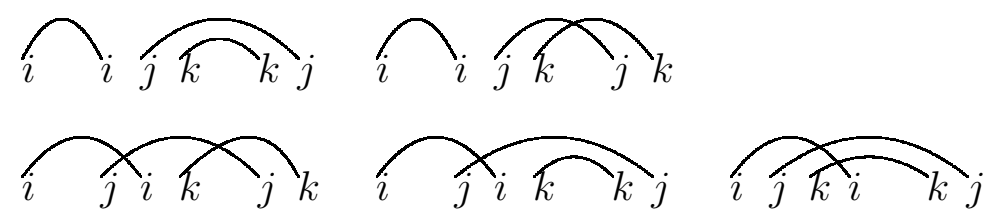

Figure 3: The forbidden patterns of three edges

Our aim is to present a decomposition of the 1132-avoiding matchings that allows us to construct the required bijection recursively. To do this, we first introduce some terminology. Let $M$ be an arbitrary matching. We call an edge $e$ of $M$ isolated if $e$ does not participate in any crossing or nesting, i.e., no edge crosses $e$, no edge is nested below $e$, and $e$ is not nested below any edge. Clearly, the isolated edges of $M$ induce in $M$ a trivial submatching. An edge that is not isolated is called important. Let $x$ be the last (i.e. rightmost) vertex of $M$ that is incident to an important edge, let $f$ be the edge incident to $x$; the edge $f$ will be called the key edge of $M$. We classify the important edges of $M$ according to their relative positions with respect to the key edge $f$ :

- An important edge is called leftist if its end point occurs before the initial point of $f$.

- An important edge is called rightist if it is nested below $f$.

- An important edge is called centrist if it crosses $f$. By the definition of $f$, such crossing necessarily occurs "from the left", i.e. the initial point of any centrist edge is to the left of the initial point of the key edge. In particular, the centrist edges form a separable submatching of $M$. 
Let $L(M), R(M), C(M)$ and $I(M)$ denote the set of leftist, rightist, centrist and isolated edges of $M$, respectively. If $M$ is trivial, then $M$ has no key edge and all its edges are isolated. In such case, we define $L(M), R(M)$ and $C(M)$ to be empty.

Observe that if $M$ is nontrivial, then $M$ must contain at least one edge that is either centrist or rightist, otherwise the key edge could not be important.

Now we are ready to describe the recursive structure of 1132-avoiding matchings.

Lemma 3.1. A matching $M$ is 1132-avoiding if and only if $M$ satisfies the following conditions:

1. The three submatchings induced respectively by the sets $L(M), R(M)$ and $C(M)$ all avoid the pattern 1132 .

2. At least one of the two sets $L(M), R(M)$ is empty.

3. If $M$ is nontrivial, then every isolated edge starts after the end point of the key edge.

4. All the leftist edges are nested below all the centrist edges.

5. All the rightist edges are nested below all the centrist edges as well.

Before we present the proof of Lemma 3.1, let us remark that the last three conditions of the lemma can be restated as follows: when we list the vertices of $M$ in the natural left-to-right order, we encounter, respectively, all the initial points of centrist edges, all the vertices incident to leftist edges, the initial point of the key edge, all the vertices incident to the rightist edges, all the end points of centrist edges, the end point of the key edge, and finally all the vertices incident to the isolated edges.

Proof of Lemma 3.1. It can be routinely checked that the five conditions are necessary. To see that they are sufficient, let $M$ be a matching that satisfies the five conditions, and assume for contradiction that $M$ contains a subsequence $i i k j$ for some $i<j<k$. Note that this implies that the edges $j$ and $k$ either cross or nest, hence the two edges are both important, and by condition (3), the edge $i$ is important as well. We distinguish the following cases:

- The edge $i$ is leftist: then there are no rightist edges in $M$ by condition (2). The edges $j, k$ cannot be centrist by condition (4), and they cannot be isolated by the argument in the previous paragraph. Hence the two edges $j, k$ are either leftist or key. However, $j$ and $k$ are nested or crossing, and if one of them is leftist, the other cannot be the key edge. This means that all the three edges $i, j, k$ are leftist, contradicting condition (1) of the lemma.

- The edge $i$ is rightist: since the two edges $j, k$ start after the edge $i$, they must be rightist as well, contradicting (1). 
- The edge $i$ is centrist: since $j, k$ end after the end point of $i$, they must be either centrist or key. It follows that the occurrences of $j$ and $k$ in the forbidden pattern $i i k j$ correspond to the end points of the two edges, hence $k$ is nested below $j$. This implies that neither $k$ nor $j$ is the key edge and we have a contradiction with (1).

- The edge $i$ is the key edge: this is impossible, because no important edge ends after the key edge.

This concludes the proof.

As a preliminary step towards the proof of the main result of this section, we prove the following theorem.

Theorem 3.2. There is a bijection $\psi$ from the set of 1132-avoiding separable matchings with $n$ edges to the set of Dyck paths of semilength $n$.

Proof. Let us proceed by induction. Of course, the empty matching is mapped to the path consisting of a single point, and the matching with a single edge is mapped to the unique Dyck path of semilength 1 . Let $M$ be a separable 1132-avoiding matching with $n>1$ edges. Notice that $M$ is nontrivial, and hence it contains a key edge. Since $M$ is separable, it does not contain any leftist or isolated edges. Let $M_{C}$ and $M_{R}$ be the submatchings of $M$ formed by the centrist and rightist edges, respectively. Clearly, both $M_{C}$ and $M_{R}$ are separable, since they are submatchings of a separable matching $M$. Together, $M_{C}$ and $M_{R}$ have $n-1$ edges. We may apply induction and define $\psi(M)=U \psi\left(M_{C}\right) D \psi\left(M_{R}\right)$. It is easy to check that this is the required bijection.

As an immediate consequence of Theorem 3.2, we have the following result.

Corollary 3.3. The number of 1132-avoiding separable matchings on $[2 n]$ is the $n$-th Catalan number.

The bijection $\psi$ constructed in Theorem 3.2 will play an auxilliary part in the proof of our main result. However, it should be pointed out that $\psi$ is not a restriction of the bijection between 1132-avoiding matchings and lattice paths that we are about to construct in our next theorem.

Theorem 3.4. There is a bijection $\phi$ from the set of 1132-avoiding matchings with $n$ edges to the set of lattice paths from $(0,0)$ to $(2 n, 0)$ starting with an up step.

Proof. We define the bijection recursively. The empty matching is mapped to the lattice path consisting of a single point.

Let $M$ be a matching with $n \geq 1$ edges, and let us assume that $\phi$ has been already defined for all 1132-avoiding matchings with less than $n$ edges. Let $r$ denote the number of isolated edges of $M$. Let $M_{C}, M_{L}$ and $M_{R}$ denote the submatchings of $M$ induced respectively by the centrist, leftist and rightist edges.

Recall that $\psi$ is the bijection mapping separable 1132-avoiding matchings to Dyck paths, as defined in the proof of Theorem 3.2. To define $\phi(M)$, we distinguish three cases: 
Case 1. $\quad M$ is trivial, i.e. $n=r$. In this case, we define

$$
\phi(M)=(U D)^{r}
$$

where $(U D)^{r}$ denotes the path consisting of $r$ consecutive occurrences of $U D$.

Case 2. $\quad M$ is nontrivial, and $M_{R}$ is nonempty. Recall that $M_{C}$ is separable, so we may apply to it the bijection $\psi$ of Theorem 3.2. We define

$$
\phi(M)=(U D)^{r} U \psi\left(M_{C}\right) \overline{D\left(M_{R}\right)}
$$

where $\overline{\phi\left(M_{R}\right)}$ denotes the mirror image of $\phi\left(M_{R}\right)$ reflected along the $x$-axis. Since $M_{L}$ must be empty whenever $M_{R}$ is nonempty, this defines a path of semilength $n$.

Case 3. $\quad M$ is nontrivial, and $M_{R}$ is empty. This implies that $M_{C}$ is nonempty, because every nontrivial matching contains either a centrist or a rightist edge. As in the previous case, we use the fact that $M_{C}$ is separable. We define

$$
\phi(M)=(U D)^{r} U \psi\left(M_{C}\right) D \phi\left(M_{L}\right) .
$$

We claim that $\phi$ is the required bijection. To see this, we need to show that $\phi$ can be inverted. Let $P$ be a lattice path of semilength $n$ starting with an up-step. There is a unique way to express $P$ as a concatenation of nonempty paths $P_{1} P_{2} \ldots P_{k}$ such that each $P_{i}$ touches the $x$-axis only at its initial point and at its end point. It follows that each of the $P_{i}$ is either a positive path (i.e., a path whose interior points are all above the $x$-axis) or a negative path. We distinguish the following cases:

- $P_{i}=U D$ for every $i \in[k]$. Then $k=n$ and we may invert the construction of case 1 above.

- There is a nonnegative integer $r \leq k-2$ such that $P_{i}=U D$ for $i \in[r], P_{r+1}$ is a positive path (possibly equal to $U D$ ) and $P_{r+2}$ is a negative path. Then we may invert the construction in the case 2 above.

- There is a nonnegative integer $r \leq k-1$ such that $P_{i}=U D$ for $i \in[r], P_{r+1}$ is a positive path different from $U D$, and either $r+1=k$ or $P_{r+2}$ is a positive path. In this situation, we invert the construction of case 3 .

It is easy to see that the cases are disjoint and cover all possibilities. This concludes the proof of the main result.

In Figure 4, we show an example of the bijection mapping a 1132-avoiding matching with 10 edges to the corresponding lattice path from $(0,0)$ to $(20,0)$. It is clear that the number of lattice paths from $(0,0)$ to $(2 n, 0)$ using steps $U=(1,1)$ and $D=(1,-1)$ starting with $(1,1)$ is counted by $\frac{1}{2}\left(\begin{array}{c}2 n \\ n\end{array}\right)$. Hence, we get the following result.

Corollary 3.5. The number of 1132-avoiding matchings on $[2 n]$ is given by $\frac{1}{2}\left(\begin{array}{c}2 n \\ n\end{array}\right)$. 


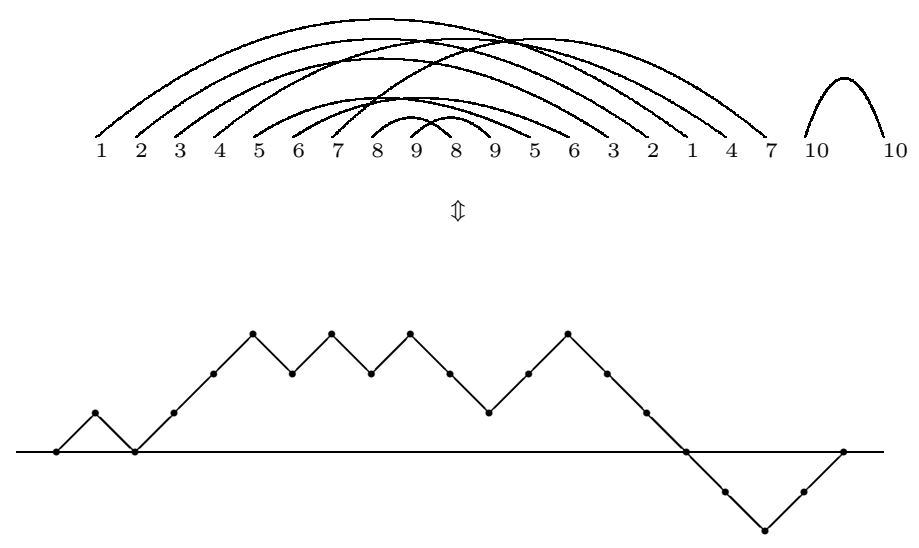

Figure 4: An example of the bijection $\phi$.

\section{References}

[1] W.Y.C. Chen, E.Y.P. Deng, R.R.X. Du, Reduction of $m$-regular noncrossing partitions, European J. Combin. 26 (2005), No. 2, 237-243.

[2] W.Y.C. Chen, E.Y.P. Deng, R.R.X. Du, R.P. Stanley, C.H.F. Yan, Crossings and nestings of matchings and partitions, ArXiv math.CO.0501230.

[3] W.Y.C. Chen, T. Mansour, S.H.F. Yan, Matchings avoiding partial patterns, ArXiv math.CO.0504342.

[4] E. Deutsch, Dyck path enumeration, Discrete Math. 204 (1999) 167-202.

[5] I.M. Gessel And X.G. Viennot, Determinants, paths and plane partitions, preprint, 1989.

[6] D. Gouyou-Beauchamps, Standard Young tableaux of height 4 and 5, European Journal of Combinatorics 10 (1989) 69-82.

[7] D. Gouyou-Beauchamps, Chemins sous-diagonanux et tableaux de Young, Combinatoire Enumerative (Montreal 1985), Lecture Notes Math. 1234 (1986) 112-125.

[8] M. Klazar, On $a b a b$-free and abba-free set partitions, European Journal of Combinatorics 17 (1996) 53-68.

[9] M. KlazAR, Bell numbers, their relatives, and algebraic differential equations, Journal Combinatorial Theory, Series A 102 (2003) 63-87.

[10] M. Klazar, Non-recursiveness of number of matchings or linear chord diagrams with many crossings, Advances in Applied Mathematics 30 (2003) 126-136.

[11] C. Krattenthaler, Permutations with restricted patterns and Dyck paths, Advances in Applied Mathematics 27 (2001) 510-530.

[12] A. DE MÉdicis And X.G. Viennot, Moments des q-polynômes de Laguerre et la bijection de Foata-Zeilberger, Advances in Applied Mathematics 15 (1994) 262-304.

[13] M. De Sainte-Catherine, Couplages et Pfaffiens en combinatoire, physique et informatique, Ph.D. Thesis, University of Bordeaux I, 1983. 
[14] R. P. Stanley, Enumerative Combinatorics, Vol. 2, Cambridge University Press, 1999.

[15] P. R. Stein, On a class of linked diagrams, I. Enumerations, Journal Combinatorial Theory, Series A 24 (1978) 357-366.

[16] J. Touchard, Sur une Problème de Configurations et sur les Fractions Continues, Canad. J. Math. 4 (1952) 2-25. 